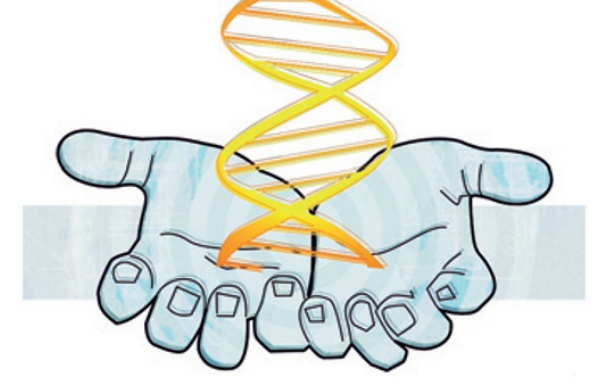

\title{
Rare diseases and now rare data?
}

\author{
Deborah Mascalzoni 1,2, Bartha Maria Knoppers ${ }^{3}$, Ségolène Aymé4 ${ }^{4}$, Matteo Macilotti ${ }^{5}$, \\ Hugh Dawkins ${ }^{6}$, Simon Woods ${ }^{7}$ and Mats G. Hansson ${ }^{2}$ \\ Rare disease research is threatened by proposed changes to European privacy laws.
}

'EURAC research, Center for Biomedicine, Bioethics, ELSI, via Galvani 31, I-39100 Bolzano, Italy.

${ }^{2}$ Centre for Research Ethics and Bioethics, Uppsala University, PO Box 564, SE-751 22 Uppsala, Sweden ${ }^{3}$ Centre of Genomics and Policy, Faculty of Medicine, Department of Human Genetics, McGill University, 740 Dr. Penfield Avenue, Room 5214, Montreal H3A OG1, Quebec, Canada.

${ }^{4}$ Rare Disease Platform, 96 rue Didot, 75014 Paris, France.

${ }^{5}$ Doctoral School in

Comparative and European Legal Studies, University of Trento, via Giuseppe Verdi 53 1-38122 Trento, Italy. ${ }^{6}$ Office of Population Health Genomics, Public Health and Clinical Services Division, Health Department of Western Australia, Level 3C, 189 Royal Street, Perth. Western Australia, Australia. ${ }^{7}$ Policy, Ethics and Life Sciences (PEALS) Research Centre, Newcastle University, 4th Floor, Claremont Bridge, Newcastle upon Tyne NE1 7RU, UK.

Correspondence to D.M. e-mail:deborah.mascalzoni@ eurac.edu doi: $10.1038 / \mathrm{nrg} 3494$

Published online 23 April 2013
The European Reform of the Data Protection Rules (RoDPR) and the Amendments proposed by the Committee on Civil Liberties, Justice and Home Affairs $(\mathrm{LIBE})^{1}$, which are currently under discussion in the European Union (EU) Parliament, have alarmed the scientific community. The declared focus of RoDPR is to strengthen privacy protection and personal control over data in general. However, by trying to apply to research the same set of rules developed for regulating commerce or the Internet, the RoDPR could be seriously detrimental to health research. Research based on biobanks and registries is effective only if access to sufficient data is granted and if the use of data is maximized through data sharing ${ }^{2}$. This is particularly important for rare disease research.

Current EU privacy rules sustain individuals' rights to manage their personal information, with consent being required for each specific use of the data. However, the requirements are more flexible for the use of data for health research: these data can be shared if they are codified and if safety measures are in place to ensure confidentiality, including ethical review. Self-determination is ensured through broad informed consent and the right to withdraw. The RoDPR foresees measures to ensure confidentiality but asks for specific consent for the use of data except for research purposes, for which an exception is granted. The LIBE Amendments propose to cancel this exception, which would seriously hinder effective data sharing.

The changes are proposed in spite of the global acceptance of the importance of open data sharing for health research, which is considered to be so compelling that data sharing has become a legal requirement for publicly funded projects in many countries. Requiring specific consent for every single use of data would have a detrimental effect on research in general and especially for rare diseases research, for which very limited data are available. The proposed revisions allow no exceptions, including secondary use of data, the use of data after death of subjects and the use of historical registries, which are so vital, especially for rare diseases. This is why the European Commission ${ }^{3}$ requires all member nations to have a rare disease research plan in place. A key element of such national plans is the development of registries, which are essential in order to capture sufficient data to make research feasible. Patient organizations led by the European Organization for Rare Diseases (EURORDIS) have expressed their right to better health and their deeply held concerns about the proposed amendments in a letter to the EU Parliament.

The Charter of Fundamental Rights of the EU recognizes the rights to integrity and protection of personal data, as well as the rights of access to preventive health care and to benefit from medical treatment. The right to privacy is not an absolute right and should be balanced with these other rights, the quality of medical treatment being a vital concern ${ }^{4}$. Other values to consider are the right to determine freely how to participate in research, which is based on the principle of autonomy, and the right to contribute samples and data in the name of solidarity.

LIBE fears that private companies will exploit the research exception to control data without informing consumers and that genetic data could be linked to individuals without consent. However, if research data are appropriately coded and if review mechanisms provide oversight on access, these risks will be minimized. Other provisions to regulate and to control data flow in research could also help to address citizens' fears ${ }^{5}$.

Rare diseases research requires participation across national borders to gain sufficient statistical significance and cannot face the loss of any collected data. Therefore, we strongly believe that the exception for scientific research should be maintained in the upcoming legislation.

\footnotetext{
LIBE. European Parliament [online], http://www.europarl.europa.eu/ meetdocs/2009_2014/documents/libe/dv/3 2012_0011. cod/32012 0011 cod en.pdf (2009).

2. Kaye, J. et al. Nature Rev. Genet. 10, 331-335 (2009).

3. Council of the European Union. europa.eu [online], http://eur-lex. europa.eu/LexUriServ/LexUriServ.do?uri = OJ:C:2009:151:0007:0 010:EN:PDF (2009).

4. Hansson, M. G. Theor. Med. Bioeth. 33, 313-323 (2012)

5. Kaye, J. Annu. Rev. Genom. Hum. Genet. 13, 415-431 (2012).
}

\section{Acknowledgements}

Research funded by the $7^{\text {th }}$ Framework EU-programs RD-Connect, EuroTEAM, BiobankCloud, BBMRI-LPC and P3G.

Competing interests statement

The authors declare no competing financial interests. 